\title{
D-Serine in Glia and Neurons Derives from 3-Phosphoglycerate Dehydrogenase
}

\author{
Jeffrey T. Ehmsen, ${ }^{1 \star}$ Ting Martin Ma, ${ }^{1 \star}$ Hagit Sason, ${ }^{4}$ Dina Rosenberg, ${ }^{4}$ Tadashi Ogo, ${ }^{5}$ Shigeki Furuya, ${ }^{5}$ \\ Solomon H. Snyder, ${ }^{1,2,3}$ and Herman Wolosker ${ }^{4}$ \\ ${ }^{1}$ The Solomon H. Snyder Department of Neuroscience, ${ }^{2}$ Department of Pharmacology and Molecular Sciences, and ${ }^{3}$ Department of Psychiatry and \\ Behavioral Sciences, The Johns Hopkins University School of Medicine, Baltimore, Maryland 21205, ${ }^{4}$ Department of Biochemistry, The Rappaport Faculty \\ of Medicine and Research Institute, Technion-Israel Institute of Technology, Haifa, 31096, Israel, and 5 Department of Bioscience and Biotechnology, \\ Graduate School of Bioresource and Bioenvironmental Sciences, Kyushu University, Fukuokashi, Fukuoka 812-8581, Japan
}

D-Serine is an endogenous ligand for NMDARs generated from L-serine by the enzyme serine racemase (Srr). Both neuronal and glial localizations have been reported for D-serine and Srr. 3-Phosphoglycerate dehydrogenase is an exclusively astrocytic enzyme that catalyzes the first committed step of L-serine biosynthesis. Using transgenic mice expressing enhanced green fluorescent protein under the Srr promoter and mice with targeted deletion of Srr or 3-Phosphoglycerate dehydrogenase, we demonstrate predominantly neuronal sources of D-serine dependent on astrocytic supply of L-serine. These findings clarify the cellular basis for the regulation of NMDAR neurotransmission by D-serine.

\section{Introduction}

Mammalian biology is stereospecific, with D-sugars and L-amino acids predominating. Recently, substantial concentrations of D-serine, D-aspartate, and, to a lesser extent, D-alanine have been identified in mammalian brain with $\mathrm{D}$-serine levels of $\sim 0.3 \mathrm{mM}$, which is one-third of L-serine levels (Hashimoto et al., 1992). Immunohistochemical localizations of D-serine resembling NMDAR distributions, in concert with the greater potency of D-serine than glycine at NMDARs, suggested a role for D-serine as an agonist at the "glycine" site of these receptors (Schell et al., 1995, 1997; Mothet et al., 2000). Selective degradation of D-serine by D-amino acid oxidase markedly reduces NMDAR neurotransmission in hippocampal preparations, establishing a physiological role for D-serine as a mediator of NMDAR transmission (Mothet et al., 2000; Papouin et al., 2012). In the hypothalamus, NMDAR transmission is reduced by D-amino acid oxidase treat-

Received 0ct. 18, 2012; revised May 21, 2013; accepted June 18, 2013.

Author contributions: J.T.E., S.H.S., and H.W. designed research; J.T.E., T.M.M., H.S., D.R., and T.0. performed research; S.F. contributed unpublished reagents/analytic tools; J.T.E., S.H.S., and H.W. analyzed data; J.T.E., T.M.M., H.S., D.R., T.O., S.F., S.H.S., and H.W. wrote the paper.

This work was supported by the National Institutes of Health (Grants \#MH18501 to S.H.S. and \#NS050275 to the MPI Microscopy Core Facility), the Israel Science Foundation, the International Mental Health Research Organization, the Legacy Heritage Fund and the R\&M Sternberg Research Fund in Alzheimer's (to H.W.), and the Japan Society for the Promotion of Science (KAKENHI Grants-in-Aid for Scientific Research Areas B \#18300125 and \#22380078 to S.F.). We thank Dr. Michael Schell for valuable suggestions, Norman Barker and Jon Christofersen for expert light microscopy, photographic, and figure assembly assistance, Dr. Richard Huganir for antibodies to GFP, and Drs. Michele Pucak and Edith Suss-Toby for expert confocal microscopy assistance.

*J.T.E. and T.M.M. contributed equally to this work.

Correspondence should be addressed to either of the following: Herman Wolosker, Department of Biochemistry, The Ruth and Bruce Rappaport Faculty of Medicine, Technion-Israel Institute of Technology, 1 Efron Street, Haifa, 31096, Israel, E-mail: hwoloske@tx.technion.ac.il; or Solomon H. Snyder, The Solomon H. Snyder Department of Neuroscience, The Johns Hopkins University School of Medicine, 725 N. Wolfe Street, Baltimore, Maryland 21205. E-mail: ssnyder@jhmi.edu.

DOI:10.1523/JNEUROSCI.4914-12.2013

Copyright $\odot 2013$ the authors $\quad 0270-6474 / 13 / 3312464-06 \$ 15.00 / 0$ ment, but not by degradation of glycine with glycine oxidase (Panatier et al., 2006). Recently, it was established that synaptic and extrasynaptic NMDAR transmission is mediated by D-serine and glycine, respectively (Papouin et al., 2012).

The biosynthesis of D-serine was clarified by the purification (Wolosker et al., 1999a) and molecular cloning (Wolosker et al., 1999b) of serine racemase (Srr), which transforms L-serine to D-serine. Initial localizations of D-serine (Schell et al., 1997) and Srr (Wolosker et al., 1999b) to astrocytes suggested that D-serine acts as a glial transmitter. More recently, however, neuronal localizations have been reported for Srr (Kartvelishvily et al., 2006; Miya et al., 2008; Benneyworth et al., 2012) and D-serine (Kartvelishvily et al., 2006); there remains no clear consensus on the cellular origin of D-serine.

L-serine synthesis in the brain occurs predominantly in astrocytes because of the virtually exclusive astrocytic localization of 3-phosphoglycerate dehydrogenase (Phgdh), which catalyzes the first committed step in L-serine biosynthesis (Yamasaki et al., 2001). The importance of Phgdh for D-serine generation is evident by decreases in brain levels of $\mathrm{D}$-serine in mice with targeted deletion of Phgdh (Yang et al., 2010).

To clarify the role of Phgdh in the formation of D-serine in glial and/or neuronal compartments, here we used mice with targeted deletion of Srr or Phgdh and improved immunohistochemical techniques for D-serine detection. We have also used $\mathrm{BAC}$ transgenic mice expressing enhanced green fluorescent protein (eGFP) under control of an Srr promoter. We describe neuronal and glial localizations of Srr and D-serine and demonstrate the dependence of D-serine in both of these compartments upon astrocytic Phgdh.

\section{Materials and Methods}

Animal husbandry. Mice containing targeted mutations of Srr and radial glia/astrocyte-specific deletion of $P h g d h$ have been described previously 
(Basu et al., 2009; Yang et al., 2010). BAC transgenic animals [Tg(SrrEGFP)KJ355Gsat/Mmucd] were obtained from the GENSAT/Mutant Mouse Regional Resource Center. Male and female mice were used equally and all studies were conducted on matched littermates. Experiments were performed in accordance with protocols approved by the animal care and use committee at Johns Hopkins University.

Protein immunofluorescence and immunohistochemistry. Immunofluorescence and immunohistochemistry were conducted as described previously (Kartvelishvily et al., 2006). Primary antibodies included: rabbit-anti-GFP (1:200; a gift from Dr. Richard Huganir), mouse antiNeuN (1:500; Millipore), guinea pig anti-glial fibrillary acidic protein (anti-GFAP, 1:500; Synaptic Systems), mouse anti-S100 $\beta$ (1:200; BD Biosciences), mouse anti-olig1 (1:200 NeuroMab), and rabbit antiPhgdh (1:500; Frontier Biosciences). Images were acquired using a Zeiss Meta 510 confocal microscope.

Amino acid immunohistochemistry. Postnatal day 16 (P16)-P21 or P56-P70 mice were deeply anesthetized with $0.4 \mathrm{mg} / \mathrm{g} \mathrm{2,2,2-}$ tribromoethanol (Avertin), and then perfused transcardially at $3 \mathrm{ml} /$ min first briefly with oxygenated buffer containing the following (in $\mathrm{mm}): 118 \mathrm{NaCl}, 4.7 \mathrm{KCl}, 2 \mathrm{CaCl}_{2}, 1.2 \mathrm{MgSO}_{4}, 1.2 \mathrm{KH}_{3} \mathrm{PO}_{4}, 25 \mathrm{NaHCO}_{3}$, and 11 glucose, followed by perfusion with freshly prepared $5 \%$ glutaraldehyde (Electron Microscopy Sciences), 0.5\% paraformaldehyde, and $0.2 \% \mathrm{Na}_{2} \mathrm{~S}_{2} \mathrm{O}_{5}$ in $0.1 \mathrm{M} \mathrm{PB}, \mathrm{pH} 7.4$, for $15 \mathrm{~min}$. After fixation and cryoprotection, free-floating sections $(20-25 \mu \mathrm{m})$ were reduced for $20 \mathrm{~min}$ in freshly prepared $0.5 \% \mathrm{NaBH}_{4}$ in TBS containing $0.2 \% \mathrm{Na}_{2} \mathrm{~S}_{2} \mathrm{O}_{5}$, washed in TBS containing $0.2 \% \mathrm{Na}_{2} \mathrm{~S}_{2} \mathrm{O}_{5}$, and affixed to gelatin-coated slides. Sections from littermates of each genotype were affixed to the same slide to ensure identical processing conditions. Slides were incubated for $24 \mathrm{~h}$ at $4^{\circ} \mathrm{C}$ with rabbit-anti-D-serine antibodies $(1: 100-1: 400$, Millipore or 1:100-1:400, Schell et al., 1995) or rabbit-anti-L-serine (1:100-1:400; Millipore) in TBS containing 4\% NGS, $0.1 \%$ Triton X-100, and $0.02 \%$ $\mathrm{NaN}_{3}$. To avoid nonspecific cross-reactivity with some tissue amino acids, incubations for D-serine detection included $0.2 \mathrm{~mm}$ glutaraldehyde conjugates of L-serine, GABA, and L-cysteine. Incubations for L-serine detection included $0.2 \mathrm{~mm}$ conjugates of D-serine, GABA, and cysteine. For Procedure A, slides were incubated for $1 \mathrm{~h}$ with a biotinylated antirabbit antibody (1:200, Vector Laboratories) in TBS containing 1.5\% NGS and $0.05 \%$ Triton X-100, washed in TBS containing $0.05 \%$ Triton $\mathrm{X}-100$, and then incubated $45 \mathrm{~min}$ in avidin-biotin-HRP solution (1:50; Vector Laboratories). For Procedure B, slides were incubated for $30 \mathrm{~min}$ with ImmPress HRP-conjugated anti-rabbit secondary antibody (Vector Laboratories). For both procedures, slides were developed for 3-7 min with one of the following substrates: SG (Vector Laboratories), DAB (Vector Laboratories), or $0.5 \mathrm{mg} / \mathrm{ml} \mathrm{DAB}, 0.008 \% \mathrm{NiCl}_{2}$, and $0.01 \%$ $\mathrm{H}_{2} \mathrm{O}_{2}$ in PBS. Sections were dehydrated in an ascending ethanol gradient, cleared in xylenes, and coverslipped using DPX mountant. Slides were visualized on a Zeiss Axiovis microscope. Semiquantification of DAB staining was performed using ImageJ software with image inversion and background subtraction (Schneider et al., 2012). For quantification of astrocytic versus neuronal D-serine labeling, astrocytes were identified by colabeling for Phgdh and neurons were identified by hematoxylin staining and nuclear morphology.

Lentiviral production. shRNA targeting murine Phgdh (V2LMM_83466) in pGipz vector and a nonsilencing control were obtained from Open Biosystems. Lentivirus was prepared essentially as described by Tiscornia et al. (2006).

Neuronal and glial culture. Primary neurons were prepared from embryonic day 15 (E15)-E16 wild-type mice as described previously (Kartvelishvily et al., 2006). At DIV, 10-12 neurons were transitioned to media lacking L-serine and glycine (BME/2\% B27 supplemented with 20 $\mu \mathrm{M}$ L-glutamine, with $1 \mathrm{~h}$ of treatment in the same media to remove any residual L-serine/glycine). Astrocytes were prepared from P0 Srr-null mice as described previously (Kartvelishvily et al., 2006) and transduced at DIV 9-13 with lentivirus harboring Phgdh-silencing or nonsilencing shRNA (multiplicity of infection, $\sim 30$ ). After $3 \mathrm{~d}$ of puromycin selection $(2 \mathrm{mg} / \mathrm{ml})$, astrocytes were washed and incubated for $96 \mathrm{~h}$ in BME/2\% B27 to generate glia-conditioned media.

Amino acid quantification. Amino acids were quantified by HPLC as described by Grant et al. (2006). To confirm peak identities and to re-
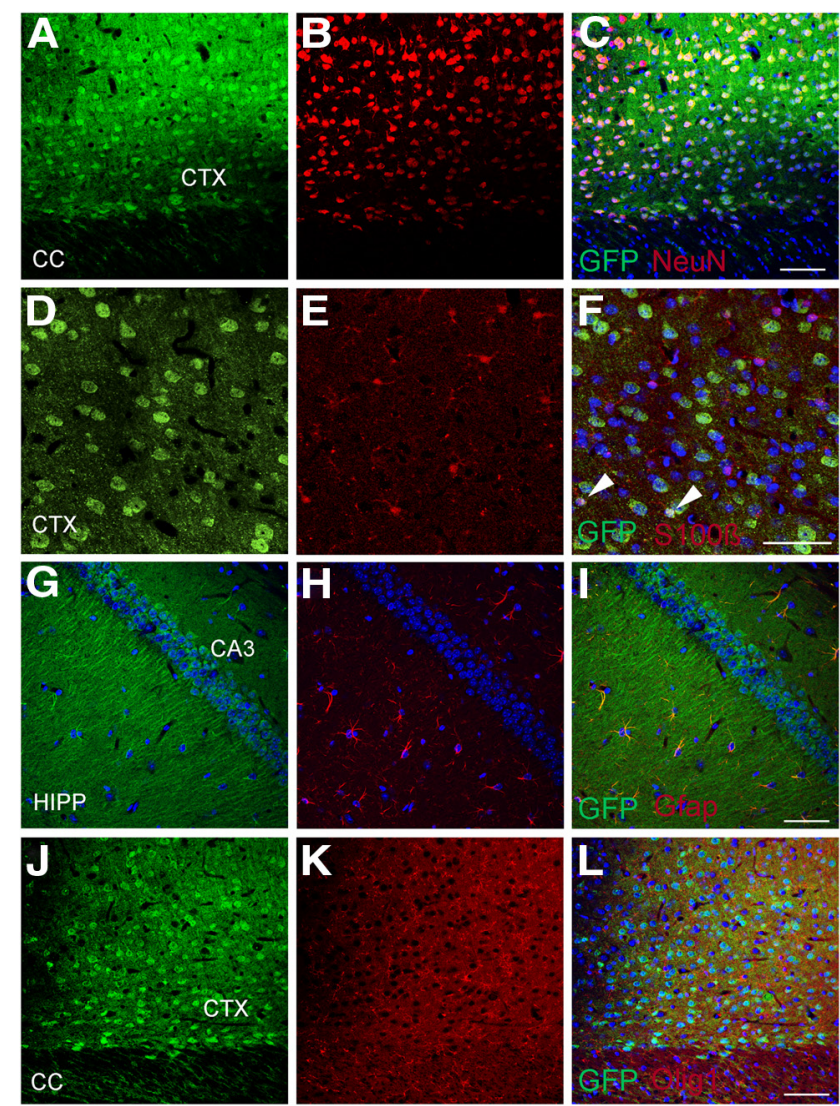

Figure 1. Serine racemase is expressed predominantly in neurons, with widespread distribution in the brain. Some glial staining is also observed. BAC-transgenic mice expressing eGFP under the control of the Srr promoter were stained for GFP and cell-specific markers. Srr expression is observed in principal neurons of the cortex (CTX, $A-C)$; shown here are layer 6 polymorphic neurons identified by neuronal nuclear (NeuN) staining. In the cerebral cortex (CTX, $\boldsymbol{D}-\boldsymbol{F}$ ), some astrocytes ( $\sim 18 \%$, identified by $\$ 100$ b staining) also appear to express low levels of Srr ( $\boldsymbol{F}$, arrowheads). Srr expression is also observed in pyramidal neurons of the entire hippocampal CA-field (HIPP, G-I). Srr expression in astrocytes, identified by staining for GFAP, is clearest in the hippocampal formation $(\mathbf{G}-\mathbf{I})$. At this level of detection, Srr is not seen in oligodendrocytes identified by staining for oligodendrocyte transcription factor 1 (olig-1) $(\boldsymbol{J}-\boldsymbol{L})$. Scale bars represent $50 \mu \mathrm{m}$.

move interfering peaks, media were pretreated with enzymes to remove L-serine (L-serine dehydratase), glutamine (L-glutaminase), or D-serine (D-serine dehydratase), as described by Radzishevsky and Wolosker (2012).

Statistical analysis. Mean pixel densities among Srr genotypes were compared using one-way ANOVA with Bonferroni correction; Mean pixel densities between Phgdh genotypes were compared by Welch's $t$ test ( $n=60$ cells for corpus callosal astrocytes and hippocampal neurons, $n=120$ for cortical neurons). Mean D-serine production by neurons cultured with GCM from control versus Phgdh-depleted astrocytes was compared using Student's $t$ test.

\section{Results}

\section{Neuronal and astrocytic localizations of serine racemase in BAC transgenic mice}

Immunohistochemically identified Srr was initially reported to be exclusively astrocytic (Wolosker et al., 1999b; Panatier et al., 2006), whereas later, using new antibodies, Srr appeared to be mainly neuronal (Kartvelishvily et al., 2006; Miya et al., 2008; Benneyworth et al., 2012). These discrepancies may reflect difficulties in selective identification of antigens by immunohistochemistry. To enhance specificity, we used BAC transgenic mice expressing eGFP under control of an Srr promoter [Tg(Srr- 
EGFP)KJ355Gsat/Mmucd] as a surrogate marker for Srr. eGFP staining is widespread and is not evident in control littermates (data not shown). We observed staining for Srr in glutamatergic principal neurons of all layers of the cerebral cortex and in the pyramidal cell layer of the hippocampal CA fields (Fig. $1 A-C, G-I$ ). To a lesser extent, we also detected Srr in astrocytes (identified by GFAP or S100 calcium binding protein $\beta[S 100 \beta])$, with staining most apparent in the hippocampal formation and cerebellar white matter (Fig. 1D-F, G-I). At our level of detection, Srr expression in oligodendrocytes, identified by staining for oligodendrocyte transcription factor 1 (Olig-1), appeared to be low or absent (Fig. $1 J-L$ ).

\section{D-Serine localized to neurons and astrocytes}

As with Srr, reports of D-serine localizations have varied with descriptions of astrocytic (Schell et al., 1997; Kartvelishvily et al., 2006; Williams et al., 2006) and neuronal (Kartvelishvily et al., 2006) sites. A limitation in assessing the specificity of immunohistochemical findings is that antigen preadsorption of antibodies as a control does not ensure specificity. The optimal and only certain control is the use of mice with genetic deletion of the corresponding antibody target. Most immunohistochemical or immunofluorescence studies of $\mathrm{D}$-serine have used biotin-dependent signal amplification (referred to here as Procedure A). The multiple amplifications inherent to this procedure can exceed the linear range of correlation between antigen quantity and signal intensity, leading to signal saturation and/or nonspecificity. We have used a more recently developed technique in which a secondary antibody is directly conjugated to polymers of HRP, which, under our experimental conditions, retains staining within a qualitatively linear range (Procedure B).

We compared Procedures A and B for the staining of D-serine in the brain using an antibody previously developed by us, as well as a commercially available antibody. Both antibodies provided similar localizations of D-serine with a broad distribution throughout the brain. Putative D-serine with Procedure A was not consistently or reliably reduced in Srr knock-out mice (Fig. $2 A, B$ ). In contrast, we observed a consistently marked reduction of labeling in $\mathrm{Sr} r$ knock-outs using Procedure B and applied this method for detailed mapping investigations (Fig. 2C,D).

We observe D-serine staining in principal neurons throughout all cortical layers, with apparent enrichment in layer 6 (Figs. 2E, 3A). Glial cells with astrocytic structure were strongly labeled in the corpus callosum (Figs. 2E, 3C). In the hippocampus, pyramidal neurons of all CA fields, especially CA3, were labeled. Scarce astrocytic labeling in the cortex and
B

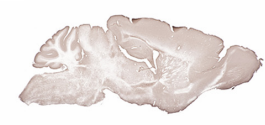

D
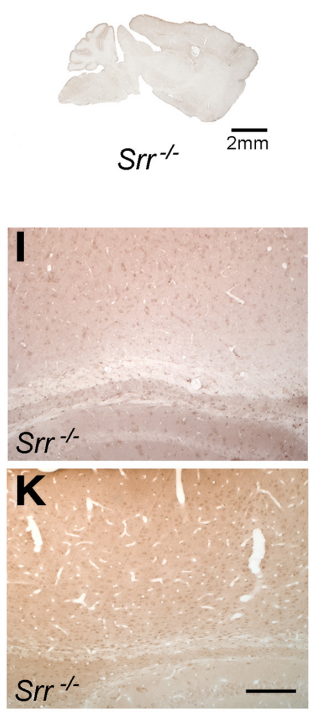

M

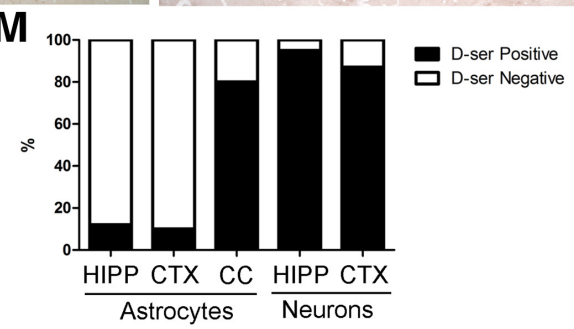

Figure 2. D-Serine is present predominantly in neurons, with restricted glial labeling. Consistent reductions in D-serine staining ot evident among Srr wild-type $(\boldsymbol{A})$ and Srr-deleted littermates $(\boldsymbol{B})$ using standard immunoperoxidase methods, but a reliable (C, D) using modified methods as described in the Materials and Methods. With these modifications, D-serine is ( mong wild-type animals, the proportion of neurons labeled is substantially larger than astrocytes $(\boldsymbol{M})$. Scale bars, $2 \mathrm{~mm}(\boldsymbol{A}-\boldsymbol{D})$, $100 \mu \mathrm{m}(\boldsymbol{E}-\boldsymbol{G})$, and $200 \mu \mathrm{m}(\boldsymbol{H}-\boldsymbol{K})$. Error bars represent mean \pm SEM.

hippocampus was detected (Figs. 2E, $3 A, E$ ). Staining in all brain regions for both neurons and astrocytes was significantly reduced in homozygous $\mathrm{Sr}$ knock-outs (Figs. $2 G, 3 B, D, F$ ). Residual staining may reflect the fact that $\sim 10-15 \%$ of D-serine levels are retained in Srr knock-out mice (Basu et al., 2009). Heterozygous Srr mutants display an intermediate reduction in staining in both neurons and glial cells (Fig. $2 F$ ); although negligible D-serine is detected in most cortical neuronal layers, moderate levels persist in layer 6, presumably reflecting higher basal levels of D-serine in these cells. Semiquantification of D-serine labeling in cortical and hippocampal neurons and astrocytes of the corpus callosum indicates a significant difference among all three genotypes $(p<0.001)$ in all three regions (Fig. $2 L$ ). Among astrocytes colabeled for Phgdh, we estimate that $\sim 10-12 \%$ of cortical and hippocampal astrocytes contain D-serine in wild-type animals. In contrast, $87-95 \%$ of hippocampal pyramidal and cortical principal neurons contain D-serine (Fig. 2M). We did not 


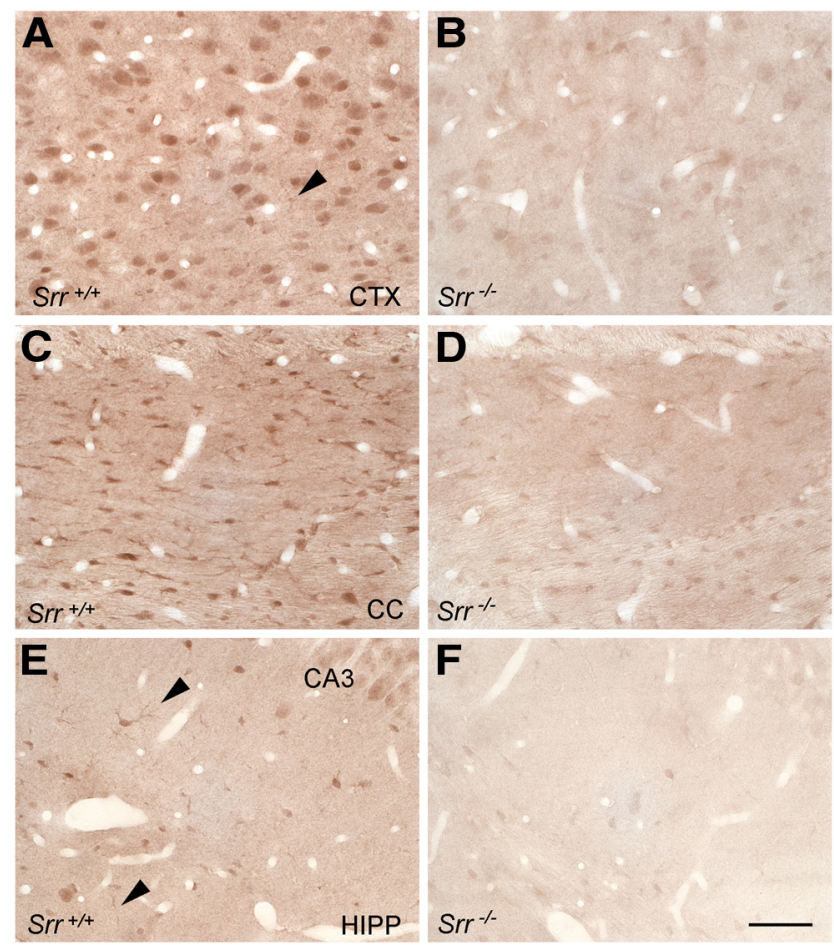

Figure 3. At higher magnification, D-serine is clearly present in polymorphic neurons of the cortex $(\boldsymbol{A})$, but low-level astrocytic labeling is also seen (arrowhead). Prominent glial staining is seen in the corpus callosum (C). Astrocytes containing D-serine are also observed in the hippocampus ( $\boldsymbol{E}$, arrowheads). D-Serine staining in both neurons and astrocytes is markedly reduced in $\mathrm{Srr}^{-1-}$ mice $(\boldsymbol{B}, \boldsymbol{D}, \boldsymbol{F})$. Scale bars, $100 \mu \mathrm{m}$.

observe overt differences in localization between young (P16P21) and adult (P56-P70) mice or between genders.

\section{Deletion of Phgdh depletes D-serine}

The large neuronal pool of D-serine we identified by immunohistochemistry seems discordant with the scarcity of L-serine available for Srr in neurons. Furuya et al. (2000) established that Phgdh, which catalyzes the first committed step in the biosynthesis of L-serine in the brain, is highly concentrated selectively in astrocytes. Accordingly, we found that primary astrocytes cultured in medium lacking L-serine or glycine accumulated L-serine in the medium (Fig. $4 A$ ); in contrast, primary cortical neurons did not produce appreciable levels of either L- or D-serine, reflecting their relative inability to generate L-serine from glucose (Fig. 4B).

To evaluate a possible role for astrocyte-derived L-serine in neuronal D-serine synthesis, we prepared Srr-null astrocytes transduced with either shRNA targeting Phgdh or a nonsilencing control. Phgdh-depleted astrocytes accumulate 20-fold less L-serine compared with controls (Fig. 4A). When incubated with conditioned medium from $S r$-null control astrocytes, neurons generated substantial amounts of D-serine (Fig. 4B). However, neurons incubated with conditioned medium from Phgdhdepleted astrocytes generated nearly 4 -fold less D-serine compared with control (Fig. $4 B ; p<0.05$ ). This effect was rescued by $\mathrm{L}$-serine supplementation, indicating that the observed reduction in neuronal D-serine synthesis results from selective depletion of L-serine from the media of Phgdh-depleted astrocytes (Fig. 4B).

The addition of glycine to primary neuronal cultures does not influence intracellular D-serine concentrations in the presence or absence of L-serine (441 pmol D-serine/mg protein in media con- taining $100 \mu \mathrm{m}$ L-serine vs $448 \mathrm{pmol} \mathrm{D}$-serine/mg protein in media containing both $100 \mu \mathrm{M}$ L-serine and $200 \mu \mathrm{M}$ glycine), suggesting that L-serine derived from glycine is unlikely to contribute significantly to neuronal D-serine production.

To verify whether Phgdh contributes to the genesis of D-serine in astrocytes and neurons in vivo, we examined immunohistochemical localizations of Phgdh and D-serine in mice in which Phgdh was deleted by GFAP-driven expression of Cre recombinase. Confirming earlier results (Yamasaki et al., 2001), we observed robust staining for Phgdh highly localized to astrocytes, prominently in the corpus callosum and throughout the cerebral cortex (Fig. 4F). This staining was virtually abolished in preparations from Phgdh-deleted mice (Fig. 4G). Correspondingly, L-serine labeling was also reduced in Phgdh knock-out brains compared with controls (Fig. 4H,I).

We compared staining of D-serine in the cortex and hippocampus of control $\left(P h g d h^{\text {flox/flox }}\right)$ and Phgdh knock-out (GFAP-Cre; $P h g d h^{\text {flox/flox })}$ brains. In the control animals, D-serine occurred in both neurons and glia with a cellular distribution identical to that described for wild-type Srr mouse models (Fig. 4D). D-Serine staining was significantly diminished in Phgdh-deleted animals in both neurons and glia (Fig. $4 E, C ; p<$ 0.0001 ). Reduction was similar in all areas of the cerebral cortex and hippocampus.

\section{Discussion}

In the present study, we establish that neuronal and glial D-serine both arise from the actions of Phgdh. Phgdh is localized virtually exclusively to astrocytes in the brain; astrocyte-derived L-serine is critical for the survival and function of neurons, enabling their synthesis of serine-derived lipids (Furuya et al., 2000). This metabolic requirement presumably underlies the profound neurologic disturbances of patients with PHGDH deficiency (de Koning et al., 2003; Kawakami et al., 2009). The loss of D-serine as a regulator of NMDAR transmission may also mediate abnormalities in PHGDH-deficient individuals.

Characterizing the role of Phgdh in generating neuronal/glial pools of D-serine required definitive clarification of the localizations of Srr and D-serine in neurons and astrocytes. We showed that reliable localizations require the use of Srr-deleted mice to establish specificity of staining. We established that D-serine is present in the principal neurons of all cortical layers and pyramidal neurons of all hippocampal CA fields. Neuronal D-serine appears to be far more substantial than was thought previously. We observed high levels of D-serine in glial cells of the corpus callosum, where Srr expression is moderate, suggesting enrichment due to high levels of Phgdh-derived L-serine within these cells.

For Srr and for D-serine, there are concerns about how best to ensure specificity of staining. Accordingly, we used mice carrying a transgene expressing eGFP under the control of an Srr promoter. Localizations of Srr in neurons and astrocytes observed with the transgenic mice resemble observations obtained by immunohistochemistry of Srr (Kartvelishvily et al., 2006; Miya et al., 2008), suggesting the validity of these localizations and confirming neuronal predominance.

D-Serine is well established as an endogenous agonist for the "glycine site" of NMDARs (Mothet et al., 2000; Shleper et al., 2005; Papouin et al., 2012). The depletion of D-serine from both astrocytes and neurons in Phgdh knock-out mice establishes astrocytes as the ultimate source for both neuronal and astrocytic pools of D-serine. These observations raise questions about the biosynthesis of $\mathrm{D}$-serine pools involved in NMDAR transmission. Our data support the inference that L-serine shuttles from astro- 
A

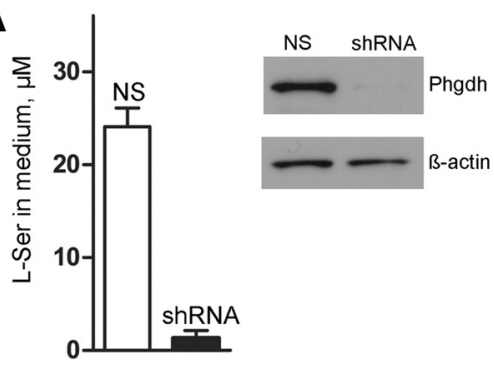

B

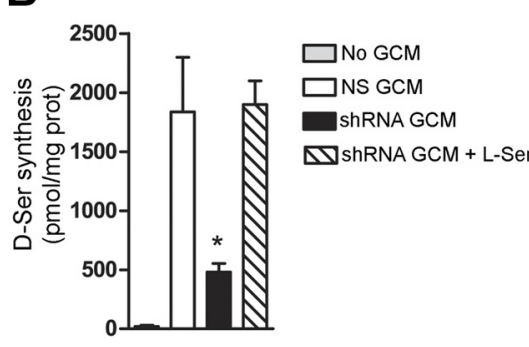

C

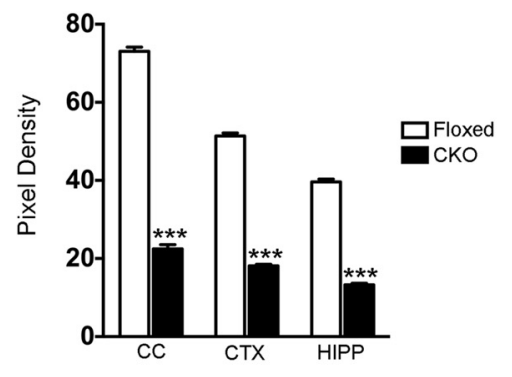

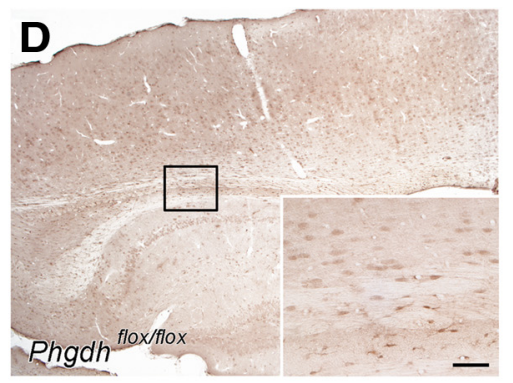

E

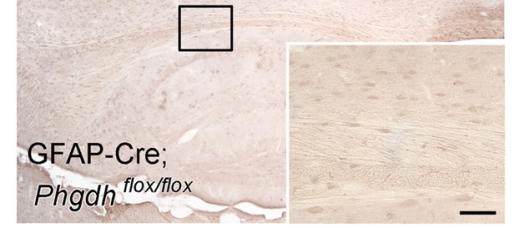

$\mathbf{F}$

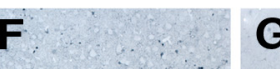

G.

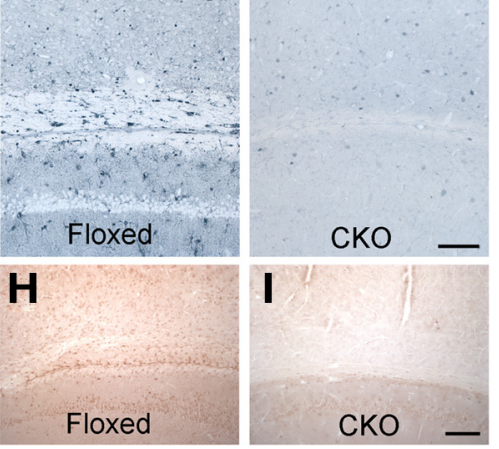

Figure 4. Role of astrocytic Phgdh for D-serine synthesis in both neurons and glia. Cultured astrocytes obtained from Srr-null mice transduced with shRNA targeting Phgdh show $>20$-fold reduction in L-serine synthesis compared with nonsilenced (NS) control astrocytes $(\boldsymbol{A})$. Western blot analysis demonstrates efficient suppression of Phgdh in shRNA-transduced astrocytes. Wildtype neurons cultured in BME/2\% B-27 alone (No GCM, where GCM is glial-conditioned media) produce at most $20 \pm 10$ pmol $\mathrm{D}$-serine/mg protein $/ 24 \mathrm{~h}(\boldsymbol{B}, n=3)$. When cultured with GCM from astrocytes transduced with a nonsilencing control shRNA (NS $G C M$ ), neuronal D-serine production increases nearly 90 -fold. Neurons cultured with GCM from Phgdh-depleted astrocytes (shRNA $G C M$ ) produce significantly less $D$-serine $\left({ }^{*} p<0.05\right)$. Supplementation with $25 \mu$ M L-serine (shRNA GCM + L-ser) restores D-serine production to levels similar to neurons cultured with control GCM. Radial glia/astrocyte-specific Phgdh-deleted mice show reduced $D$-serine levels in both glia and neurons $(\boldsymbol{D}, \boldsymbol{E})$. In Phgdh ${ }^{\text {flox/flox }}$ mice, D-serine is detected in neurons and glia with widespread distribution (D). D-serine levels in both cell types are significantly reduced in GFAP-Cre;Phgdh ${ }^{\text {flox/flox }}$ mice $(\boldsymbol{E})$, demonstrating a requirement of astrocytic Phgdh activity for D-serine synthesis in both cell types. Semiquantification of D-serine labeling indicates a significant difference between Phgdh ${ }^{\text {flox/flox }}$ (floxed) and GFAP-Cre;Phgdh ${ }^{\text {flox/flox }}$ (CKO) mice (C, $\left.{ }^{* * *} p<0.0001\right)$. Phgdh is enriched in astrocytes in Phgdh ${ }^{\text {flox/flox }}$ mice $(\boldsymbol{F})$, with efficiently targeted deletion of the enzyme in GFAP-Cre; Phgdh ${ }^{\text {flox/flox }}$ littermates $(\boldsymbol{G})$. L-Serine in GFAP-Cre; Phgdh ${ }^{\text {flox/flox }}$ mice is notably reduced $(\boldsymbol{I})$ compared with littermate controls $(\boldsymbol{H})$. Scale bars, $50 \mu \mathrm{m}$ $(\boldsymbol{D}-\boldsymbol{G})$ and $100 \mu \mathrm{m}(\boldsymbol{H}, \boldsymbol{I})$. Error bars represent mean \pm SEM.

cytes into neurons, where it is transformed by neuronal Srr to D-serine; this appears to be a principal route of D-serine synthesis.

GFAP-driven expression of Cre recombinase as a tool for glial-specific gene excision is widely used, but may be limited by variations in specificity of expression and/or efficiency (Sofroniew, 2012). Recent studies have indicated that GFAP is expressed in early radial glia, including those that give rise to some neurons and oligodendrocytes (Casper and McCarthy, 2006). Although the possibility of some neuronal recombination during development cannot be excluded, the observation that Phgdh expression is restricted to astrocytes in the adult mouse CNS makes these concerns unlikely to change the interpretation of our findings. In addition, the specific Cre line used in this study shows high recombination efficiency in astrocytes, as demonstrated by nearly complete absence of Phgdh expression from these cells in adult GFAP-Cre; $P h g d h^{\text {flox/flox }}$ animals (Fig. $4 F, G)$.

The conclusion that synthesis of both neuronal and astrocytic D-serine is dependent on Phgdh may have therapeutic relevance. A substantial portion of neurotoxicity associated with vascular strokes is thought to reflect massive release of glutamate activating NMDARs, because NMDA antagonists reduce stroke damage in animal models (Kalia et al., 2008). Correspondingly, neurotoxicity associated with ischemia elicited by middle cerebral artery occlusion is reduced in Srr knock-out mice (Mustafa et al., 2010). The depletion of D-serine associated with Phgdh deletion is similar in magnitude to that after Srr deletion (Basu et al., 2009; Yang et al., 2010). Therefore, PHGDH inhibitors might offer benefit in attenuating neurotoxicity in vascular stroke and other diseases involving neurotoxicity linked to NMDAR overactivation. Although genetic impairment of PHGDH activity is associated with neurologic disability, transient treatment with PHGDH inhibitors may not be deleterious.

\section{References}

Basu AC, Tsai GE, Ma CL, Ehmsen JT, Mustafa AK, Han L, Jiang ZI, Benneyworth MA, Froimowitz MP, Lange N, Snyder SH, Bergeron R, Coyle JT (2009) Targeted disruption of serine racemase affects glutamatergic neurotransmission and behavior. Mol Psychiatry 14:719-727. CrossRef Medline

Benneyworth MA, Li Y, Basu AC, Bolshakov VY, Coyle JT (2012) Cell selective conditional null mutants of serine racemase demonstrate a predominate localization in cortical glutamatergic neurons. Cell Mol Neurobiol 32: 613-624. CrossRef Medline

Casper KB, McCarthy KD (2006) GFAP-positive progenitor cells produce neurons and oligodendrocytes throughout the CNS. Mol Cell Neurosci 31:676-684. CrossRef Medline

de Koning TJ, Snell K, Duran M, Berger R, PollThe BT, Surtees R (2003) L-serine in disease and development. Biochem J 371:653-661. CrossRef Medline

Furuya S, Tabata T, Mitoma J, Yamada K, Yamasaki M, Makino A, Yamamoto T, Watanabe M, Kano M, Hirabayashi Y (2000) L-serine and glycine serve as major astroglia-derived trophic factors for cerebellar Purkinje neurons. Proc Natl Acad Sci U S A 97:11528-11533. CrossRef Medline

Grant SL, Shulman Y, Tibbo P, Hampson DR, Baker GB (2006) Determination of D-serine and related neuroactive amino acids in human plasma by high-performance liquid chromatography with fluorimetric detection. J Chromatogr B Analyt Technol Biomed Life Sci 844:278-282. CrossRef Medline

Hashimoto A, Nishikawa T, Oka T, Takahashi K, Hayashi T (1992) Determination of free amino acid enantiomers in rat brain and serum by high-performance liquid chromatography after derivatization with $\mathrm{N}$-tert.-butyloxycarbonyl-L-cysteine and $o$-phthaldialdehyde. J Chromatogr 582:41-48. Medline 
Kalia LV, Kalia SK, Salter MW (2008) NMDA receptors in clinical neurology: excitatory times ahead. Lancet Neurol 7:742-755. CrossRef Medline

Kartvelishvily E, Shleper M, Balan L, Dumin E, Wolosker H (2006) Neuronderived D-serine release provides a novel means to activate $N$-methyl-Daspartate receptors. J Biol Chem 281:14151-14162. CrossRef Medline

Kawakami Y, Yoshida K, Yang JH, Suzuki T, Azuma N, Sakai K, Hashikawa T, Watanabe M, Yasuda K, Kuhara S, Hirabayashi Y, Furuya S (2009) Impaired neurogenesis in embryonic spinal cord of Phgdh knockout mice, a serine deficiency disorder model. Neurosci Res 63:184-193. CrossRef Medline

Miya K, Inoue R, Takata Y, Abe M, Natsume R, Sakimura K, Hongou K, Miyawaki T, Mori H (2008) Serine racemase is predominantly localized in neurons in mouse brain. J Comp Neurol 510:641-654. CrossRef Medline

Mothet JP, Parent AT, Wolosker H, Brady RO Jr, Linden DJ, Ferris CD, Rogawski MA, Snyder SH (2000) D-serine is an endogenous ligand for the glycine site of the $N$-methyl-D-aspartate receptor. Proc Natl Acad Sci U S A 97:4926-4931. CrossRef Medline

Mustafa AK, Ahmad AS, Zeynalov E, Gazi SK, Sikka G, Ehmsen JT, Barrow RK, Coyle JT, Snyder SH, Doré S (2010) Serine racemase deletion protects against cerebral ischemia and excitotoxicity. J Neurosci 30:1413-1416. CrossRef Medline

Panatier A, Theodosis DT, Mothet JP, Touquet B, Pollegioni L, Poulain DA, Oliet SH (2006) Glia-derived D-serine controls NMDA receptor activity and synaptic memory. Cell 125:775-784. CrossRef Medline

Papouin T, Ladépêche L, Ruel J, Sacchi S, Labasque M, Hanini M, Groc L, Pollegioni L, Mothet JP, Oliet SH (2012) Synaptic and extrasynaptic NMDA receptors are gated by different endogenous coagonists. Cell 150: 633-646. CrossRef Medline

Radzishevsky I, Wolosker H (2012) An enzymatic-HPLC assay to monitor endogenous D-serine release from neuronal cultures. Methods Mol Biol 794:291-297. CrossRef Medline

Schell MJ, Molliver ME, Snyder SH (1995) D-serine, an endogenous synaptic modulator: localization to astrocytes and glutamate-stimulated release. Proc Natl Acad Sci U S A 92:3948-3952. CrossRef Medline

Schell MJ, Brady RO Jr, Molliver ME, Snyder SH (1997) D-serine as a neu- romodulator: regional and developmental localizations in rat brain glia resemble NMDA receptors. J Neurosci 17:1604-1615. Medline

Schneider CA, Rasband WS, Eliceiri KW (2012) NIH Image to ImageJ: 25 years of image analysis. Nat Methods 9:671-675. CrossRef Medline

Shleper M, Kartvelishvily E, Wolosker H (2005) D-serine is the dominant endogenous coagonist for NMDA receptor neurotoxicity in organotypic hippocampal slices. J Neurosci 25:9413-9417. CrossRef Medline

Sofroniew MV (2012) Transgenic techniques for cell ablation or molecular deletion to investigate functions of astrocytes and other GFAP-expressing cell types. Methods Mol Biol 814:531-544. CrossRef Medline

Tiscornia G, Singer O, Verma IM (2006) Production and purification of lentiviral vectors. Nat Protoc 1:241-245. CrossRef Medline

Williams SM, Diaz CM, Macnab LT, Sullivan RK, Pow DV (2006) Immunocytochemical analysis of D-serine distribution in the mammalian brain reveals novel anatomical compartmentalizations in glia and neurons. Glia 53:401-411. CrossRef Medline

Wolosker H, Blackshaw S, Snyder SH (1999a) Serine racemase: a glial enzyme synthesizing D-serine to regulate glutamate- $N$-methyl-D-aspartate neurotransmission. Proc Natl Acad Sci U S A 96:13409-13414. CrossRef Medline

Wolosker H, Sheth KN, Takahashi M, Mothet JP, Brady RO Jr, Ferris CD, Snyder SH (1999b) Purification of serine racemase: biosynthesis of the neuromodulator D-serine. Proc Natl Acad Sci U S A 96:721-725. CrossRef Medline

Yamasaki M, Yamada K, Furuya S, Mitoma J, Hirabayashi Y, Watanabe M (2001) 3-Phosphoglycerate dehydrogenase, a key enzyme for L-serine biosynthesis, is preferentially expressed in the radial glia/astrocyte lineage and olfactory ensheathing glia in the mouse brain. J Neurosci 21:7691-7704. Medline

Yang JH, Wada A, Yoshida K, Miyoshi Y, Sayano T, Esaki K, Kinoshita MO, Tomonaga S, Azuma N, Watanabe M, Hamase K, Zaitsu K, Machida T, Messing A, Itohara S, Hirabayashi Y, Furuya S (2010) Brain-specific Phgdh deletion reveals a pivotal role for L-serine biosynthesis in controlling the level of D-serine, an $\mathrm{N}$-methyl-D-aspartate receptor coagonist, in adult brain. J Biol Chem 285:41380-41390. CrossRef Medline 